\title{
A Critical Gap: \\ In situ Measurements of Planetary Surface-Atmosphere Interactions Beyond Earth
}

\author{
Corresponding Author: Serina Diniega (Jet Propulsion Laboratory, California Institute of \\ Technology; serina.diniega@jpl.nasa.gov; 626-720-7293)
}

Co-authors: Devon Burr (N Arizona U), Colin M. Dundas (U.S. Geological Survey), Brian Jackson (Boise State U), Michael Mischna (JPL), Scot Rafkin (SwRI), Isaac Smith (York U + PSI), Robert Sullivan (Cornell U), Timothy Titus (U.S. Geological Survey), Nathalie Vriend (University of Cambridge), Ian Walker (Arizona State U), Kaj Williams (U.S. Geological Survey)

Acknowledgements: A portion of the work described was carried out at the Jet Propulsion Laboratory, California Institute of Technology, under a contract with the National Aeronautics and Space Administration (80NM0018D0004).

Signatories: Shane Byrne (U Arizona), Matt Chojnacki (PSI), Ken Cooper (JPL), Ingrid Daubar (Brown U), Mackenzie Day (UCLA), Cynthia L. Dinwiddie (SwRI), Francesca Esposito (INAF, Italy), Vic Etyemezian (Desert Res Inst), Lori Fenton (SETI Inst), Clémence Herny (U Bern), Devanshu Jha (MVJ College of Engineering, India), Laura Kerber (JPL), Aditya Khuller (ASU), Tim Michaels (SETI), Claire Newman (Aeolis Research), Alejandro Soto (SwRI), Christy Swann (Naval Res Lab), Leslie Tamppari (JPL), Jim Zimbelman (Smithsonian Inst)

\section{Executive Summary}

This white paper demonstrates five points: (1) The lack of robust measurements of the vertical gradients of natural boundary layers and transport fluxes on other planetary bodies precludes adequate estimation of aeolian and other meteorological processes throughout our Solar System (§1). (2) Thus, there exist critical knowledge gaps within high-priority planetary science questions that motivate the need for in situ aeolian and other meteorological measurements on an extraterrestrial surface (§2). (3) Such measurements would be timely, because they will greatly enhance the utility of existing climate data/models, while also contributing to improved design, safety, and effectiveness of near-future missions (§3). (4) Acquisition of these nextgeneration measurements from Mars is technologically feasible within the coming decade (including via small spacecraft) $(\S 4,6)$ and $(5)$ such measurements would also significantly contribute towards addressing key Mars science questions (§5). Our implicit recommendation is that this type of in situ science be considered in definition of high-priority planetary science questions and prioritization of mission concepts.

(C) 2020. All rights reserved.

Submitted to the Planetary Science \& Astrobiology Decadal Survey, 2023-2032 


\begin{abstract}
1. Importance of Surface-Atmosphere Interactions in Planetary Science
Surface ${ }^{*}$-atmosphere interactions, resulting in the exchange of volatiles and transport of sediments (sand and dust) and volatiles, are key active processes on all rocky planetary bodies, even those hosting only a transient and/or thin "atmosphere." These interactions help shape planetary landscapes, affect the sources/sinks of crucial atmospheric constituents, and influence climatological and meteorological dynamics. All of these connections affect interpretation of observed or modeled geology and climate, identification of potentially habitable environments, and our understanding of a planetary body's history (Table 1). Terrestrial processes are intensively studied via numerical modeling that integrates the results from laboratory studies and field investigations of driving conditions and resultant activity, along with measurement of the relevant landforms. This incorporation of in situ measurements has resulted in robust testing and calibration/refinement of the surface-atmosphere interaction process models, in Earth conditions. Such testing and refinement have, so far, not been possible in other planetary environments.
\end{abstract}

Table 1: Some high-priority planetary studies that reply upon surface-atmosphere interaction process models.

\begin{tabular}{|c|c|}
\hline Models & es \\
\hline \multirow{6}{*}{$\begin{array}{l}\text { Aeolian processes create bedforms } \\
\text { (e.g., ripples and dunes), shape } \\
\text { landscapes via erosion, and deposit } \\
\text { material that creates sedimentary rock } \\
\text { records, providing clues to past } \\
\text { environments. } \\
\text { Lacking a tested quantitative model } \\
\text { of how to connect specific atmospheric } \\
\text { (e.g., pressure, wind speeds) and } \\
\text { surface conditions to the erosion and } \\
\text { transport of dust and sand brings } \\
\text { uncertainty into... }\end{array}$} & $\begin{array}{l}\ldots \text { the sources/amounts of aerosols }- \text { shown to be key } \\
\text { inputs in climate modeling in general and including } \\
\text { generation of large dust storms (e.g., Mars, Titan, Earth). }\end{array}$ \\
\hline & $\begin{array}{l}\text {... estimations of exhumation rates, which are important for } \\
\text { biosignature detection and sampling strategies (e.g., } \\
\left.\text { Mars }^{1} \text { ), potential release of volatiles (e.g., Mars }{ }^{2}\right) \text { as well as }\end{array}$ \\
\hline & $\begin{array}{l}\text { interpretation of excavated landscapes (e.g., Gale Crater, } \\
\text { Mars }^{3} \text { ). }\end{array}$ \\
\hline & $\begin{array}{l}\text { ion studies regarding the source and } \\
\text { al hazards }\left(e . g ., \text { Mars }^{4,5}\right) \text {. }\end{array}$ \\
\hline & $\begin{array}{l}\text { unique records of } \\
\text { in the present or } \\
\text { ial and temporal } \\
\text { luto, a comet }{ }^{6} \text { ). }\end{array}$ \\
\hline & n operations (e.g., Ma \\
\hline \multirow{4}{*}{$\begin{array}{l}\text { The rates and methods of } \\
\text { atmosphere-surface or -regolith } \\
\text { exchange of volatiles are important } \\
\text { controls on surface activity, distribution } \\
\text { and preservation of subsurface water } \\
\text { ice, crucial unknowns within models of } \\
\text { sublimation landforms (e.g., } \\
\text { thermokarst), and contributors to the } \\
\text { atmospheric system and related } \\
\text { processes. }\end{array}$} & $\begin{array}{l}\text { W. estir } \\
\text { - often } \\
\text { and for }\end{array}$ \\
\hline & $\begin{array}{l}\ldots \text { dete } \\
\text { to dete } \\
\text { may bi } \\
\end{array}$ \\
\hline & $\begin{array}{r}\text { sits - } \\
\text { past } \\
\end{array}$ \\
\hline & \\
\hline \multirow{2}{*}{$\begin{array}{l}\text { Lacking a tested quantitative model } \\
\text { to connect specific environmental } \\
\text { conditions to the surface volatile flux } \\
\text { brings uncertainty into ... }\end{array}$} & $\begin{array}{l}\text { face ice and implications for the } \\
\left.\text {., } \text { Ceres }^{8}, \text { Vesta }^{9}, \text { Mars }^{10}, \text { Pluto }^{11}\right) \text {. }\end{array}$ \\
\hline & past or present habitability on local- \\
\hline
\end{tabular}

For example, sand grain sizes in martian dunes remained uncertain until Curiosity's in situ exploration of a dune field, which addressed a mismatch of orbital thermal inertia models predicting relatively coarse grains vs. wind tunnel studies indicating finer grains would be easier

\footnotetext{
* Throughout this white paper, we focus on interactions between atmospheres and solid surfaces.
} 
to transport. Until the Mars Exploration Rovers saw sand moving in situ and the Mars Reconnaissance Orbiter tracked ripple and dune migration from orbit, it was not clear that sand transport and bedform evolution was occurring in the present martian climate 6 . Fundamental uncertainties remain about how factors like atmospheric density and wind speed affect dust lofting, sand saltation, and the development of bedforms - on Mars and other planetary bodies (see §2, 5 for more details). Filling critical gaps in the universal physics of surface-atmosphere interactions requires in situ measurements on a planetary body (other than Earth) that relate atmospheric dynamics to volatile fluxes and particle mobilization, transport, and deposition.

\section{Open Key Planetary Science and Fundamental Physics Questions}

The majority of our current understandings of surface-atmosphere processes have been developed for terrestrial conditions. These evolving principles can be tested for their universality by acquiring basic in situ measurements from different planetary environments. Fundamental questions that remain unaddressed for all planetary bodies (except possibly Earth) include:

- What are the minimum wind speeds that will mobilize sand and dust? How do these thresholds vary with local conditions (e.g., surface roughness, substrate cohesion, humidity, slope)?

- How does the sand saltation profile (i.e., flux and grain sizes as a function of height above ground) depend on atmospheric density and gravity?

- What is the frequency of sand and dust transport on other planetary bodies?

- By what mechanisms (e.g., wind shear, vertical vortices) is dust lofted into suspension? How much dust is lofted by each mechanism and to what heights? (In particular, what causes a convective vortex to lift dust? InSight has detected many convective vortices and dust appears available on the Mars surface, but they have not seen dust devils.)

- What regolith characteristics and environmental conditions control surface-atmosphere volatile exchanges?

High-resolution, high-frequency measurements of surface-atmosphere interactions on other planetary bodies would enable addressing questions such as those above, enabling testing and refinement of our current models and advancing development of cross-planet process models [see white paper (WP): Diniega et al.]. In particular, we could:

- Broaden knowledge of aeolian sediment transport physics by documenting it, in detail, in a vastly different density atmosphere: Extraterrestrial in situ "field" measurements of atmospheric boundary layer dynamics driving sand/dust and volatile transport would provide novel calibration data for models and wind-tunnel experiments. Such "ground truth" is needed to advance a cross-planet model for, e.g., sand and dust transport [also WP: Newman et al.]. Refining these aeolian process models would extend the return of numerous missions that have made observations of atmosphere-solid surface interactions and bedforms, and inform selected missions that seek to explore worlds with such interactions (e.g., Dragonfly).

- Test near-surface atmospheric and meteorological dynamics models: A key example of a model untested outside of Earth is the one used to estimate turbulent eddy fluxes (which result in the exchange of energy, momentum, and quantities like dust, water, and other chemical species between the surface and atmosphere). On Earth, turbulent fluxes can be directly calculated from correlated, high frequency measurements of the 3D wind components and the quantity of interest; such fluxes are also related to large-scale (and more easily measured) quantities such as the vertical gradient of temperature and the vertical gradient of the horizontal wind (i.e., the wind shear). Terrestrial field studies have been critical in highlighting, and reducing, uncertainty in using these theoretical relationships in natural boundary layers where conditions are not idealized and vertical gradients in turbulent fluctuations often deviate from model predictions ${ }^{12}$. These same relationships are assumed in planetary studies, but have never 
been shown to extend to those environments despite those environments generally being far outside of terrestrial conditions (for example, Mars has an extremely stable nocturnal inversion and unstable afternoon convective layer).

- Develop regolith- and surface-atmosphere exchange models with respect to volatiles, including the flux rates and feedback mechanisms between trace gases and surface properties: On many worlds, sublimation of subsurface ice is thought to form landforms, such as pits (e.g., Pluto ${ }^{11}$ ) and scalloped terrain (e.g., Mars ${ }^{10}$ ), as well as exospheres (e.g., Ceres ${ }^{13}$ ). Within the martian mid-latitudes, large reservoirs of near-surface ice are thought to be stabilized under an insulating, desiccated coating of dust/regolith, but how that ice got there, how long it has been preserved, and what sort of climate record it may contain are not known yet. In addition to ice, regolith can harbor adsorbed water, brine, pre-melted liquids at mineral-ice and ice-grain interfaces, or hydrous minerals (bound molecular $\mathrm{H}_{2} \mathrm{O}$ ) - all of which have the potential to exchange $\mathrm{H}_{2} \mathrm{O}$ with the atmosphere via processes partially dependent on local relative humidity and temperature conditions. Interpretations of sublimation thermokarst landforms or buried ice deposits as climate records, estimation of the lag needed to preserve ice (also of relevance for human exploration ${ }^{4,14}$ ), or estimation of the water available to induce mineralogical alteration or visible surface change (e.g., martian RSL ${ }^{15}$ ) are reliant upon models of regolith-atmosphere exchange processes and rates that remain untested within an extraterrestrial environment.

\section{The Critical Gap: Planetary In Situ Aeolian and Other Meteorological Measurements}

In situ measurements of sediment lofting/entrainment and transport and volatile atmosphereregolith exchange (including surface water ice/vapor phase changes and transport), with concurrent measurements of potential meteorological drivers and geologic environmental controls, are needed to constrain models of the fundamental physical processes involved with surfaceatmosphere interactions at the grain and landform scales. Such measurements are generally achieved on Earth via aeolian and meteorological field studies (Figure 1).

In this white paper, we start from an analogous "field investigation" approach and focus on measurements that could be made by a single landed station. Having just one aeolian/ meteorological station to acquire a full suite of in situ measurements would present a huge science advancement. Sending multiple small stations ${ }^{\dagger}$ could enable $\sim$ simultaneous collection of distributed measurements to, e.g., investigate the spatiotemporal variability of sediment transport during passage of a storm front or map the wind field and trace gases through a crater's topography. Our list of needed measurements (Table 2) has been derived from multiple discussions with terrestrial and planetary science experts focused on (1) planetary science climate and surface process investigations based on models, observations, and wind tunnel/laboratory experiments; (2) terrestrial field studies that are connected with process models; (3) collection of in situ data by Mars rovers; and (4) aeolian and meteorological instrument developers. It also builds on decades of discussion within the planetary atmospheric and aeolian communities and recent communitybased studies that outline priorities (most recently the MEPAG-chartered Ice and Climate Evolution Science Analysis Group ${ }^{16}$, WP: Newman et al.). The highest priority in situ measurements for a given planetary target would be those expected to change through diurnal and seasonal trends, or during discrete events that drive volatile/sediment flux (e.g., a large wind gust).

\footnotetext{
${ }^{\dagger}$ A slightly different set of investigations and related measurements may be prioritized with a network of lander/rover/orbiter(s) for an integrative study of a specific region (such as through a crater or full atmospheric profile) or to gather a "global" look at surface meteorological and aeolian conditions (e.g., as in the PMCS MOSAIC mission concept or NF3 concept from ICE-SAG ${ }^{16}$ ).
} 
Figure 1: Decades of terrestrial aeolian and meteorological field studies have yielded robust instrument deployment and measurement collection methodologies, which can be used to optimize analogous planetary studies. Shown here are dust/sand/wind sensors (in fore- \& background clusters) and an instrumented mast (background), deployed at Pismo Beach, CA, to determine the rate and sources of dust emissions. Similar methodologies and instruments could be deployed on another planetary surface to address the same question. Image provided by lan Walker (ASU).

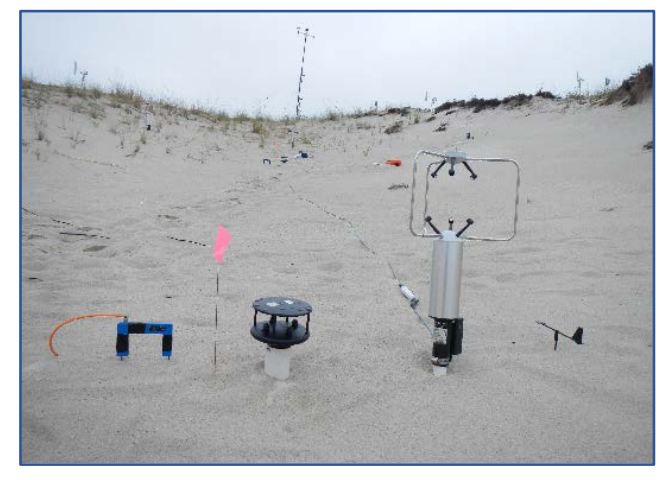

Key operational considerations include that (1) investigation-related measurements should be collected close together in time and space, as even small differences could obfuscate physical correlations (e.g., between an atmospheric eddy and upwelling of water vapor); (2) observations need to be sufficiently frequent to detect and characterize key "events," such as a large wind gust or passing dust devil; and (3) accommodation of the instruments should be carefully designed as the spacecraft can easily perturb the environment of interest-such as creating a thermal plume, blocking sand transport, or diverting the local winds.

\section{Why Mars is the Suggested Target}

We suggest that Mars is a prime target for acquiring these needed in situ observations in the next decade, for six reasons. First, Mars' atmospheric, surface, and planetary conditions are different enough from Earth's to test and stretch terrestrial study-based models, but similar enough that the terrestrial models are a reasonable starting point. Second, Mars has recognized wide-spread sediment transport and seasonal and diurnal volatile cycles [WP: Dundas et al.; Newman et al.] as well as a strong role of dust within climate variability [WP: Newman et al.]. Many volatile-driven processes and related landforms have also been identified, and in many cases, these are tied to present-day activity [WP: Dundas et al.] or to recent climate conditions driven by Mars' wellstudied obliquity cycles [WP: Becerra et al., Smith et al.].

Third, decades of previous investment have yielded ample geologic and atmospheric contextual information and models that will greatly enhance the ability to interpret new in situ measurements within the broader geologic and atmospheric/climatological context, including:

- Global imaging of aeolian bedforms (from orbit: measure many dunes and ripples ( $>1 \mathrm{~m}$ wavelength), and from rovers: observe smaller ripples and dunes, for a few locations) and some migration rates;

- Maps of areas where near-subsurface ice is likely (based on geomorphological markers, visible exposure, or subsurface sounding), and a qualitative understanding of climate cycles that would lead to redistribution of ice and mobile sediments;

- Maps of areas with present-day seasonal and diurnal frosts (and in some cases discrimination between types of frost and/or abundance estimates) and maps of related landforms;

- Global/seasonal wind patterns and a coarse understanding of what wind speeds are possible and that move sediment (from extensive modeling and limited in situ measurements);

- Measurements and models of upper atmosphere broadscale dynamics and aerosol/water content, through seasons and a few Mars years;

- Observations of dust devils, dust storms, and surface/atmospheric dust distribution; and

- Extensive datasets and experience in characterizing a specific landing area's surface topography, composition, and surface roughness - enabling in situ measurements to be placed into the local, regional, and global geologic context.

However, existing in situ aeolian and other meteorological data are insufficient to robustly answer 
Table 2: Aeolian and meteorological information needed to fill critical gaps.

\begin{tabular}{|c|c|c|}
\hline \multicolumn{2}{|c|}{ Science Investigation } & In situ Measurements \\
\hline \multirow{8}{*}{$\begin{array}{l}\text { Responses } \\
\text { Measure the } \\
\text { surface- } \\
\text { atmosphere } \\
\text { fluxes of sand, } \\
\text { dust, volatiles, } \\
\text { heat, and } \\
\text { momentum. }\end{array}$} & Volatiles & Absolute concentration \\
\hline & \multirow[t]{2}{*}{ Dust } & Local surface dust erosion \& deposition rates \\
\hline & & Lofted dust flux and grain sizes \\
\hline & \multirow[t]{2}{*}{ Sand } & Surface sand erosion \& deposition rates \\
\hline & & $\begin{array}{l}\text { Saltation profile: Number and sizes of grains in motion, as a function } \\
\text { of height }\end{array}$ \\
\hline & \multirow[t]{2}{*}{ | } & Temperature profile \\
\hline & & Net downwelling \& upwelling radiation \\
\hline & Momentum & $\begin{array}{l}\text { Horizontal wind measurements from at least three heights - to derive } \\
\text { surface shear stress; of frequency to determine "average" } \\
\text { velocities and the gust velocity distribution }\end{array}$ \\
\hline \multirow{9}{*}{$\begin{array}{l}\text { Drivers } \\
\text { Determine the } \\
\text { controls on } \\
\text { mechanisms } \\
\text { that lead to } \\
\text { sediment, } \\
\text { volatiles, and } \\
\text { heat being } \\
\text { moved from } \\
\text { the surface } \\
\text { into the } \\
\text { atmosphere \& } \\
\text { transported. }\end{array}$} & \multirow{5}{*}{$\begin{array}{l}\text { Meteorologi } \\
\text { cal Controls } \\
\text { (+ winds, } \\
\text { above) }\end{array}$} & $\begin{array}{l}\text { Atmospheric temperature and pressure - to derive atmospheric } \\
\text { density }\end{array}$ \\
\hline & & Atmospheric composition (including trace gases/humidity) \\
\hline & & $\begin{array}{l}\text { Surface pressure and temperature } \\
\text { Turbulence (i.e.. high-frequency 3D wind measurements) }\end{array}$ \\
\hline & & $\begin{array}{l}\text { Vortices/dust devils: number/frequency, surface shear stress, and } \\
\text { amount of dust carried }\end{array}$ \\
\hline & & Overhead clouds, coverage, characteristics, and altitude \\
\hline & \multirow{4}{*}{$\begin{array}{l}\text { Surface } \\
\text { Controls }\end{array}$} & Grain size distribution on the near-by surface \\
\hline & & Grain properties (e.g., angularity, composition, electrostatics) \\
\hline & & $\begin{array}{l}\text { Local surface topography, geology type (e.g., bedrock exposure, } \\
\text { dust cover), and surface roughness elements }\end{array}$ \\
\hline & & $\begin{array}{l}\text { Local surface mechanical and thermoconductive properties (e.g., } \\
\text { cohesion, thermal inertia) }\end{array}$ \\
\hline
\end{tabular}

surface-atmosphere interaction questions as no dedicated sediment sensors were included in past missions and the meteorological instruments flown were not well accommodated or designed to be part of a comprehensive aeolian/meteorological experiment ${ }^{4,16}$. Recent community-based reports ${ }^{16,17}$ have highlighted the continued need for such measurements, in particular wind and water vapor fluxes from the surface and up through the planetary boundary layer (PBL) on Mars, and show that such measurements could potentially be achieved via a small spacecraft.

Fourth, the past and current focus on Mars science and human exploration has yielded important advancements in relevant mission and instrument technologies (discussed further in §6). Fifth, commercial and international interest in sending spacecraft to Mars along with Mars' relatively close proximity to Earth suggest that ample access opportunities will exist over the next decade, especially for small spacecraft that do not need a dedicated launch vehicle.

Sixth, accomplishment of such a mission in the near-term would enhance interpretation of in situ Titan observations by Dragonfly as well as orbital and in situ data from ongoing and past planetary missions that have mapped wind- and sublimation-driven landforms and monitored atmospheric/climate and surface activity [WP: Diniega et al.]. In addition to direct observations of surface-atmosphere interactions on two planets (Earth and Mars), we also would then have in situ information from three planetary bodies with a range of atmospheric densities (low to high: Mars, Earth, and Titan). Comparative analyses of relevant landforms, atmospheric and surface conditions, and evidence of surface activity on these three worlds would enable very robust testing of cross-planet surface-atmosphere interaction models. 


\section{Open Key Mars Science Questions}

In addition to the above Planetary Science high-priority questions (§2), an advancement in our understanding of how surface and atmosphere conditions and processes generate sediment and volatile flux on Mars would contribute significantly to several areas in Mars science, including:

- Understanding how atmospheric processes have and continue to influence the observed sedimentary record and the Mars surface: On Mars, numerous observations and studies of extensive surface sediments and sedimentary deposits have demonstrated the important role of the atmosphere in Mars' sedimentary cycle. Wind-blown sand forms dunes and ripples and, as an agent of abrasion, erodes bedrock and creates dust; saltating sand also lofts dust. Windblown dust can be transported high into the atmosphere and which mantles much of the martian surface. Ancient bedrock reveals that wind-blown sand dunes and sand sheets left deposits across a water-influenced landscape ${ }^{18}$. Our ability to examine ancient sedimentary rocks on Mars and possible evidence of habitable environments [WP: Horgan et al.], especially those in which the geomorphic expression of the paleoenvironment has been etched from the surface (e.g., Jezero and Eberswalde Craters), depends on identification of how sediments were deposited and estimation of erosion by sand abrasion. In particular, determining the abrasion rates and the ultimate fate of vast amounts of material eroded from the landscape, such as occurred at Gale Crater to reveal the MSL exploration site ${ }^{3}$ has profound implications for sampling strategies for preserved biosignatures ${ }^{1}$.

- Understanding diurnal, seasonal, and annual dust/water cycles on Mars: The dust, water, and $\mathrm{CO}_{2}$ cycles are major drivers of the present climate on Mars. To better understand the radiative effects of clouds or how atmospherically deposited dust affects formation of seasonal frost, monitoring of surface and meteorological conditions is needed (ideally with global observations of clouds and the aerosol and water vapor atmospheric profile). A more robust understanding of how local-scale events lift dust from the surface and into the PBL is needed to understand how storms develop and how dust influences atmospheric circulation and vice versa [WP: Newman et al.]. A clearer understanding of how the dust and water cycles are driven and how they interact in the present atmosphere will enable atmospheric models to be refined, allowing for more confident modeling of recent past and ancient climates.

- Enhance reliability of payload delivery to surface targets and safety of future robotic and human surface operations: Any mission to the martian surface incurs risk due to our limited ability (compared to Earth) to predict atmospheric and surface conditions. Key knowledge is meteorological conditions from the surface to the top of the PBL. Increased understanding of diurnal and seasonal variability, under both ambient and dust storm conditions, of atmospheric wind speeds and temperature could be critical for future design of engineering solutions, such as aerobraking technologies. Measuring winds, temperature, humidity, and ice/dust aerosols at the surface-ideally concurrently with higher-altitude measurements, but at least able to be combined with existing global, high-altitude data—addresses strategic knowledge gaps as these would validate/improve meteorological models and promote advanced designs for entry, descent and landing (EDL) and surface operation systems. Additionally, characterization of the sizes, shapes, electrical/thermal conductivity, and heights achieved by wind-transported particles is important to assess the hazard potential of lofted grains to humans or machinery ${ }^{4}$.

\section{Existing or Maturing Technologies that Enable Mars Measurement Acquisition}

Technological advances in delivery of payloads, instruments, data processing, and data collection methodology have occurred across multiple areas that make acquisition of the needed measurements very feasible in the next decade: 
- Options for delivering small, focused, stationary payloads economically to the martian surface are in development and appear technologically feasible [WP: Barba et al.]. Such technologies could enable a dedicated spacecraft for aeolian/meteorological in situ investigation, thus alleviating accommodation concerns and operational complexity attendant with past large Mars landed spacecraft. Aeolian and meteorological investigations involve much environmental monitoring, which does not require spacecraft mobility. (Although at least some small amount of initial mobility could be highly useful so as to optimally place sensors relative to e.g., the local wind field.) Alternatively, useful investigation may be acquired on a semimobile platform. For example, a helicopter platform [WP: Bapst et al.] would provide the opportunity to do vertical profiling in the lower atmosphere at multiple locations.

- Over the last few decades, there have been large advances in miniaturization, data quality, and acquisition capabilities within relevant terrestrial field instrumentation: e.g., small and lowcost laser particle counters ${ }^{19}$ to measure grain sizes and heights as well as flux and large advancements in memory storage and onboard processing. A number of instruments, specifically designed for planetary aeolian and meteorological investigations, have also been proposed (and some are currently in-development via e.g., PICASSO): e.g., an open-path tunable laser spectrometer ${ }^{20}$ for measuring atmospheric composition, a differential absorption radar for absolute humidity measurements ${ }^{21}$, a sonic anemometer for $3 \mathrm{D}$ winds ${ }^{22}$, an optical device for measuring grain sizes/velocities in the sand flux ${ }^{23}$, a wind $\operatorname{LIDAR}^{24}$, and a net radiometer to determine the surface energy balance ${ }^{25}$.

In summary: Collecting in situ measurements to quantitatively investigate surface-atmosphere interactions on a planet other than Earth is a unique opportunity for significant advancement in numerous fundamental physics and Planetary Science questions with even a single, potentially small, new mission. Observations that directly connect environmental drivers and sediment/volatile fluxes would fill critical understanding gaps that feed into interpretations of planetary geologic and climate histories, potential for habitability, and planetary protection and mission operations design. Such data has been instrumental in terrestrial studies of analogous processes, and has been requested by the planetary aeolian and meteorological communities for decades - but no planetary mission focused on these investigations has yet flown. Mars is a promising target of such investigations due to context provided by past studies, as well as new Mars access and instrument technologies enabling measurement acquisition in the next decade. Such a mission would enhance the science contributions of existing and future missions - in particular, those studying Earth, Mars, Titan, Venus, and small icy bodies.

References (see the non-compressed list here): [1] Chojnacki et al. 2018, JGR 123, 468-88. [2] Safi et al. 2019, Sci Reports 9, 8229. [3] Anderson \& Day 2017, Phys Rev E 96, 043110. [4] MEPAG Goals, 2020. [5] NASA Planetary Protection Independent Review Board (PPIRB) Final Report, 2019. [6] Diniega et al. 2017, Aeolian Res 26, 5-27. [7] Dundas et al. 2018, Science 359, 199-201. [8] Sizemore et al. 2019, JGR 124, 1650-89. [9] Denevi et al. 2012, Science 338, 246-49. [10] Dundas et al. 2015, Icarus 262, 154-169. [11] Moore et al. 2017, Icarus 287, 320-33. [12] Li et al. 2010, GRL 37, L15404. [13] Tu et al. 2014, P\&SS 104, 157-62. [14] Mars Water In-Situ Resource Utilization (ISRU) Planning (M-WIP) Study Final Report, 2016. [15] Gough et al. 2019, Icarus 321, 1-13. [16] Ice and Climate Evolution Science Analysis Group (ICE-SAG) Final Report, 2019. [17] Next Mars Orbiter Science Analysis Group (NEX-SAG) Final Report, 2015. [18] Grotzinger et al. 2015, Science 350, aac7575. [19] Hugenholtz \& Barchyn 2011, JGR 116, F01010. [20] Rafkin 2015, IEEE Conf. [21] Cooper et al. 2020, Passive/Active mm-Wave Imaging XXIII, 1141102. [22] Banfield et al. 2016, J Acoustical Soc Am 140, 1420-8. [23] Etyemezian et al. 2017, Aeolian Res 24, 65-79. [24] Määttänen et al. 2018, 28 ${ }^{\text {th }}$ Internat Laser Radar Conf, 06006. [25] Soto et al. 2013, EPSC, 793. 